\title{
Self-organized Fission Control Algorithm for Flocking System with Time Delay
}

\author{
Panpan Yang ${ }^{1}$, Maode Yan $^{1}$ and Xiaokang Lei ${ }^{2}$ \\ 1. School of Electronic and Control Engineering, Chang 'an University, Xi'an 710064, \\ China; \\ 2. School of Information and Control Engineering, Xi'an University of Architecture \& \\ Technology, Xi'an 710055, China; \\ yangpanpan1985@126.com,mdyan@chd.edu.cn,ray_com@163.com
}

\begin{abstract}
The self-organized fission control problem for flocking system with time delay is studied in this paper. Firstly, the basic fission mechanism inspired by the splitting phenomena of biological flocks is addressed and an information coupling degree (ICD) based fission strategy as well as a pairwise interaction rule is developed. Then, by taking the time delay effect into consideration, a self-organized fission control algorithm that integrates a pairwise interaction rule is proposed, which realizes the spontaneous splitting of a cohesive flock into multiple subgroups under the effect of multiple conflict external stimuli. Finally, numerical simulations are provided to demonstrate the effectiveness of the proposed algorithm.
\end{abstract}

Keywords: Flocking system; fission control; time delay; information coupling degree; pairwise interaction

\section{Introduction}

The fission behavior of flocking system is the splitting phenomenon that a cohesive flock segregates into multiple sub-clusters [1]. It is widely observed in nature, e.g., a flock of birds or a school of fish may split to evade the attack from predators [2], a herd of sheep can break up into smaller foraging groups for grass eating on the prairie [1] and so forth. In engineering applications, fission behavior is also of great significance in such occasions as obstacle/ danger avoidance for swarm UAVs (unmanned aerial vehicles) [3], multi-site surveillance for mobile sensor networks [4] and multi-target tracking for mobile robot groups [5].

In the above cases, members in the flock are often identical ones with limited sensing and communicating capabilities, their motions only rely on the local interaction with the nearest neighbors [6]. Therefore, the fission phenomenon is virtually an emergent behavior that arises in a self-organized fashion [7][8]. How a cohesive flock splits and forms clusters remains a fascinating issue of both theoretical and practical interests.

At present, literatures that specifically address the fission behavior of flocking system seem diverse. Morgan et al. [9] proposed a homotopy control scheme that realized the splitting and merging of flocking system with leader-following dynamics. Kumar et al. [10] considered the splitting problem of heterogeneous swarm robots using different artificial potential functions. Chen et al. [11] studied the aggregation and splitting problem of flocking system via a medium range repulsion force. In addition, some multi-target tracking algorithms can also achieve the fission behavior by predefining the corresponding objectives to individuals [5][12][13]. However, these works either depend on explicit identification [10] or 
intelligent coordination mechanisms such as negotiation, appointment or centralized control [12], which may not be well consistent with the characteristics of fission behavior. To this end, Liu et al. [8] recently proposed a bio-inspired fission control algorithm that realized the splitting of a cohesive flock into multiple subgroups in a self-organized fashion.

However, these results are obtained without considering the time delay effect in the fission process. As a matter of fact, time delay is the inherent phenomenon that widely exists in the fission control of flocking system (e.g., the delay caused by finite speeds of information transmission and spreading as well as the inherent delay of sensors or actuators [14]). The presence of time delay decays the system performance and may even cause the instability of flocking system [15]. Currently, time delay problem is mostly considered in the consensus seeking or formation keeping issues. For example, Olfati-Saber et al. [16] discussed the consensus problem of multi-agent system via a frequency domain approach. Using LyapunovRazumikhin functions, Hu et al. [17] studied the consensus problem with time delays in term of LMIs. Munz et al. [18] derived a delay-independent criteria that achieved rendezvous for any constant time delay. Yang et al. [19] studied the flocking of multi-agents with time delay in both leader free and virtual leader cases. To the best of our knowledge, literatures that specifically address the time delay problem in the fission control of flocking system are rare.

In this paper, we investigate the self-organized fission control problem for flocking system with time delay. Based on the bio-inspired information coupling degree (ICD) fission strategy, a pairwise interaction rule is developed and a self-organized fission control algorithm with time delay is proposed by integrating the pairwise interaction rule into the "separation/ alignment/cohesion" flocking framework. Theoretical analysis proves that the algorithm is able to realize the fission control in the presence of time delay and numerical simulations also illustrate the effectiveness of the proposed method.

The rest of this paper is organized as follows: in Section 2, the fission control problem with time delay is addressed. In Section 3, the bio-inspired fission principle is investigated and an information coupling degree based fission strategy is developed. In section 4 , the fission control algorithm with time delay is proposed. In section 5, the main results and theoretical analysis are given. In Section 6, numerical simulations are provided to demonstrate the effectiveness of the proposed algorithm. Section 7 offers the concluding remarks and future work.

\section{Problem Formulation}

Consider a flocking system consisting of $N$ identical individuals in $n$-dimensional Euclidean space with the following dynamics

$$
\left\{\begin{array}{l}
\dot{p}_{i}=q_{i} \\
\dot{q}_{i}=u_{i}, i=1,2, \cdots, N
\end{array}\right.
$$

where $p_{i}, q_{i} \in \square^{n}$ are the position vector and velocity vector of individual $i$ and $u_{i} \in \square^{n}$ is the control input (acceleration vector) acting on it. The neighbors of individual $i$ are represented by the neighboring set $N_{i}=\left\{j:\left\|p_{j}-p_{i}\right\| \leq R, j \neq i, j=1,2, \cdots, N\right\}$, where $R$ is the sensing range of each individual.

Essentially speaking, fission behavior is an emergent phenomenon of large numbers of individuals by interacting with its neighbors and the surrounding environment [1][20]. Correspondingly, the control input acting on an individual can be written as

$$
u_{i}=u_{i}^{\text {in }}+g_{i} u_{i}^{\text {out }}
$$


where $u_{i}^{\text {in }}$ is the internal interaction force between individual $i$ and its neighbors while $u_{i}^{\text {out }}$ is the force acting on individuals from external environment. Moreover, for the limited sensibility of individuals, we let $g_{i}$ denote whether individual $i$ can sense environment information, where $g_{i}=1$ means the environment information can be directly obtained by individual $i$ and $g_{i}=0$, otherwise.

Due to the self-organized characteristic of fission behavior, environment information can only be seen as the trigger event, whether the whole flock has the ability to split is determined by its local interaction rules [8]. Therefore, the main objective of fission control is to design the distributed local interaction rules and synthesize the control input ${ }^{u_{i}}$ for a flocking system such that when conflict environment stimuli act on a small portion of individuals, the flock can segregate into clustered sub-groups spontaneously. In particular, if time delay $\tau$ exists in the fission control of flocking system, we have the following definition:

Definition 1 A flocking system (with time delay $\tau$ existing in the local interaction with its neighbors) is said to be segregated or a fission behavior occurs if it satisfies the following conditions:

(1) The distance between individuals in the same subgroup is bounded, that is,

$$
\lim _{x \rightarrow \infty}\left\|p_{i}(t-\tau)-p_{j}(t-\tau)\right\| \leq \delta, i, j \in G_{k}
$$

where $\delta>0$ is a fixed value and $G_{k}$ denotes the subgroup $k$.

(2) The velocities of individuals in the same subgroups will asymptotically converge to the same value, that is,

$$
\lim _{x \rightarrow \infty}\left\|q_{i}(t-\tau)-q_{j}(t-\tau)\right\|=0, i, j \in G_{k}
$$

(3) For individuals in different subgroups, the distance between individuals will ultimately exceed their sensing range $R$, that is,

$$
\lim _{x \rightarrow \infty}\left\|p_{i}(t-\tau)-p_{j}(t-\tau)\right\|>R, i \in G_{k}, j \in G_{s}
$$

where $G_{s}$ denotes the subgroup $s$, it means that the subgroups will lose connections with each other and the fission behavior emerges.

\section{Bio-inspired Fission Control Method}

As has been suggested by biologists, fission behavior is the spontaneous response of a cohesive flock to conflict external stimuli [21], during which the mutual interaction intensity between individuals plays a key role [22]. Individuals with larger interaction intensity tend to form clusters in the presence of significant differences in the preference of individuals [7].

Here, we define the interaction intensity between individuals as Information Coupling Degree (ICD). In biological flocking systems, individuals that directly sense external stimuli often modulate the stimulus information into their movement variations [23] and other individuals are usually very sensitive to such movement variations of their neighbors like the abrupt turning or acceleration [14]. Hence, we adopt the variation of relative position in a small time interval to formulate the information coupling degree between individual $i$ and $j$ as

$$
c_{i j}=\frac{\left\|p_{i j}^{t}-p_{i j}^{t-\Delta t}\right\|}{\left\|p_{i j}^{t}\right\|}, j \in N_{i}{ }_{i} \bigcap N_{i}{ }^{t-\Delta t}
$$

where $p_{i j}^{t}=p_{i}^{t}-p_{j}^{t}$ and $p_{i j}^{t-\Delta t}=p_{i}^{t-\Delta t}-p_{j}^{t-\Delta t}$ are the relative position between individual $i$ and $j$ at time $t$ and $t-\Delta t, \Delta t>0$ is the sampling time interval. 
From the information transfer perspective, fission behavior can be generalized as the directional information propagation process among members when a flock encounters multiple conflict stimuli [23]. In order to maximize such information transfer, we take the neighbor with the largest information coupling degree as the most correlated neighbor and propose a "maximum-ICD" based "pairwise interaction" rule in the fission control of flocking system, which is formulated as

$$
f_{i}=\left\{j \mid \max c_{i j}, c_{i j} \geq c^{*}, j \in N_{i}\right\}
$$

where $c^{*}$ is the threshold value of fission behavior, a relative large $c^{*}$ is used to prevent the unexpected segregation due to fluctuation or other unknown factors.

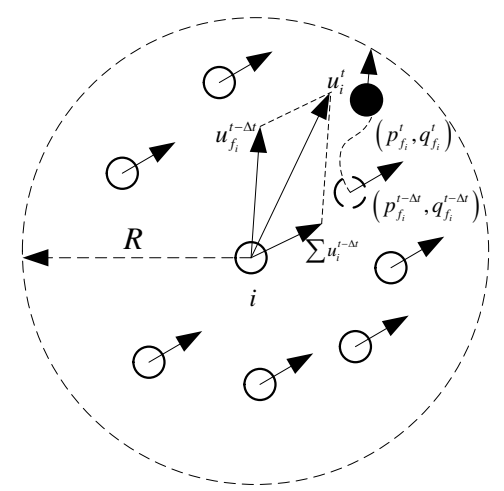

Figure 1. Internal Interaction Mechanisms of Individuals based on "MaximumICD" Strategy

The internal interaction mechanism of individuals during the fission process is illustrated in Figure 1 Assume that at time $t-\Delta t$, individual $f_{i}$ (locates at $p_{f_{i}}^{t-\Delta t}$ with velocity $q_{f_{i}}^{t-\Delta t}$ ) is propelled by external stimuli and changes its motion rapidly to a new position $p_{f_{i}}^{t}$ with velocity $q_{f_{i}}^{t}$ in a small time interval $\Delta t$. According to (3), individual $i$ is more influenced by $f_{i}$ and tends to have a relatively tighter correlation with it. Therefore, at time $t$ the force of neighbors acting on individual $i$ can be written as

$$
u_{i}^{t}=u_{f_{i}}^{t-\Delta t}+\sum_{i=1}^{N_{i}-1} u_{i}^{t-\Delta t}
$$

where $u_{f_{i}}^{t-\Delta t}$ is the pairwise interaction force of the most correlated neighbor $f_{i}$ acting on individual $i$ and $\sum_{i=1}^{N_{i}-1} u_{i}^{t-\Delta t}$ denotes the sum of the forces of other neighbors acting on it, with $N_{i}$ being the number of neighbors.

Remark 1 In (4), the most correlated neighbor of individual $i$ is dynamically changing according to (3) in every time step and the role of which is to maximize the stimuli information transfer within the flock. Thus, there is an essential difference between our work and [4][5][12], as the latter assumes the (virtual) leader or target is predefined or invariant during the evolution of the flocking system. 


\section{Fission Control Algorithm with Time Delay}

Based on the above fission method, we consider the self-organized fission control problem of flocking system with time delay. By using the proposed "pairwise interaction" rule and together with the traditional "separation/alignment/repulsion" rules [25], the fission control algorithm with time delay $\tau$ is designed as

$$
u_{i}=\underbrace{f_{i}^{p}(t-\tau)+f_{i}^{v}(t-\tau)+f_{i}^{f}(t-\tau)}_{u_{i}^{\mathrm{in}}}+g_{i} f_{i}^{e}
$$

where $u_{i}^{\text {out }}$ represents the environment force that is generated by the external stimulus $f_{i}^{e}$, it causes the rapid maneuvering of individuals and lead to the fission behavior.

Usually, there are many different forms of $f_{i}^{e}$ due to different environment stimuli, such as the direction to a known food source or a segment of migration route [7]. Here, for the ease of presentation, we take the external stimuli in the simple velocity feedback form

$$
f_{i}^{e}=-\left(q_{i}-q_{i}^{e}\right)
$$

where $q_{i}^{e}$ is the desired velocity driven by external stimuli.

Remark 2 Most of the time, individuals that sense environmental stimuli can directly response to it without time delay or the delay is much smaller compared with the information transmission delay from its neighbors [15], therefore, in (7) we ignore the time delay from external environment and only consider the time delay effect between individuals.

In addition, we can easily see that the internal interaction force $u_{i}^{\text {in }}$ consists of three components, where

(1) $f_{i}^{p}(t-\tau)$ is the position coordination term that regulates the relative positions of individuals. It includes a set of attraction/repulsion artificial potential forces that make individuals move together if individuals are too far away from each other, and propel them away to avoid collision if individuals are getting too close. A general realization of such function can be written as

$$
f_{i}^{p}(t-\tau)=-\sum_{j=1}^{N_{i}} \nabla_{p_{i j}} \varphi_{i j}\left(\left\|\left[p_{i}(t-\tau)-p_{f_{i}}(t-\tau)\right]-\left[p_{j}(t-\tau)-p_{f_{j}}(t-\tau)\right]\right\|_{\sigma}\right)
$$

where $\nabla$ is the gradient operator, ${ }^{N_{i}}$ is the number of neighbors of individual $i$, the $\sigma_{\text {-norm }}$ \|\|$_{\sigma}$ of a vector is a map $\square^{n} \rightarrow \square^{+}$defined by $\|z\|_{\sigma}=(1 / \varepsilon)\left[\sqrt{1+\varepsilon\|z\|^{2}}-1\right]$ with a parameter $\varepsilon>0$ and $z=\left[z_{1}, z_{2}, \cdots, z_{n}\right]^{\mathrm{T}} \in \square^{n}$. Note that $\|z\|_{\sigma}$ is differentiable everywhere, but $\|z\|=\sqrt{z_{1}^{2}+z_{2}^{2}+\cdots+z_{n}^{2}}$ is not differentiable at $z=0$. This property of $\sigma$-norm is used for the construction of smooth collective potential functions for individuals. The construction of a smooth pairwise potential with finite cut-off can be referred to [25].

(2) $f_{i}^{v}(t-\tau)$ is the velocity regulation term that coordinates the velocities of individuals

$$
f_{i}^{v}(t-\tau)=-\sum_{j=1}^{N_{i}} a_{i j}\left(\left[q_{i}(t-\tau)-q_{f_{i}}(t-\tau)\right]-\left[q_{j}(t-\tau)-q_{f_{j}}(t-\tau)\right]\right)
$$

where $a_{i j}$ is the element of adjacency matrix $A$ and its definition can be referred to [19]. 
(3) $f_{i}^{f}(t-\tau)$ is the pairwise interaction term with its most correlated neighbors, both position and velocity feedbacks are used to make the movement of individual $i$ converge to that of $f_{i}$

$$
f_{i}(t-\tau)=-k_{1}\left(p_{i}(t-\tau)-p_{f_{i}}(t-\tau)\right)-k_{2}\left(q_{i}(t-\tau)-q_{f_{i}}(t-\tau)\right)
$$

where $k_{1}, k_{2}>0$ are feedback gains to be assigned. This term guarantees the convergence of individual $i$ to its most correlated neighbor $f_{i}$ and induces the segregation of the flock if conflict information exists in the flock.

As the environment stimuli is only the trigger event of fission behavior and the external force acting on individuals is often much bigger than that of the internal force, therefore, individuals that are propelled by external stimuli can be assumed to be affected by external force only [7]. In the later analysis, we will neglect the influence of environment stimuli and only consider the internal interaction force $u_{i}^{\text {in }}$ if no confusion arises.

Let $\tilde{p}_{i}=p_{i}-p_{f_{i}}, \tilde{q}_{i}=q_{i}-q_{f_{i}}$ be the position error and velocity error between individual $i$ and its most correlated neighbor $f_{i}$, respectively. Then, we can get the control input in the error form

$$
\begin{aligned}
u_{i}= & -\sum_{j=1}^{N_{i}} \nabla_{p_{i j}} \tilde{\varphi}_{i j}\left(\left\|\tilde{p}_{i}(t-\tau)-\tilde{p}_{j}(t-\tau)\right\|_{\sigma}\right)-\sum_{j=1}^{N_{i}} a_{i j}\left(\tilde{q}_{i}(t-\tau)-\tilde{q}_{j}(t-\tau)\right) \\
& -k_{1} \tilde{p}_{i}(t-\tau)-k_{2} \tilde{q}_{i}(t-\tau)
\end{aligned}
$$

In the following section, we will give some results and theoretical analysis of our proposed algorithm.

\section{The Main Theoretical Results}

Here, we define the following Lyapunov function:

$$
\begin{aligned}
Q= & k_{1} \sum_{i=1}^{N_{i}} \tilde{p}_{i}^{T}(t-\tau) \tilde{p}_{i}(t-\tau)+\sum_{i=1}^{N_{i}} \tilde{q}_{i}^{T}(t-\tau) \tilde{q}_{i}(t-\tau) \\
& +\sum_{i=1}^{N_{i}} \sum_{j=1}^{N_{i}} a_{i j} \int_{\tau}^{t} \tilde{q}_{i}^{T}(t-\eta) \tilde{q}_{i}(t-\eta) d \eta+\sum_{i=1}^{N_{i}} \sum_{j=1}^{N_{i}} a_{i j} \tilde{\varphi}_{i j}
\end{aligned}
$$

Obviously, $Q$ is a positive semi-definite function. We have the following results.

Theorem 1 Consider a flocking system governed by dynamics (1) and with time delay $\tau$, assume the initial graph is connected, the initial energy $Q_{0}\left(Q_{0}=Q(\tilde{p}(0), \tilde{q}(0))\right)$ of the subgroup is finite and the initial velocity error is zero, i.e., $\tilde{q}_{i}(0)=0$. Then, when conflict external stimuli act on partial members in the flock, a cohesive flock will split into clustered subgroups under the fission control algorithm (6) and individuals in the same subgroup will attain the same velocity asymptotically.

Proof We take a cluster of $N_{i}$ individuals in the neighborhood of individual $i$ into consideration and assume the graph $G_{N_{i}}$ is connected in the small sampling time interval from $t$ to $t+\Delta t$.

Take the derivative of (17), it obtains 


$$
\begin{aligned}
\dot{Q}= & 2 k_{1} \sum_{i=1}^{N_{i}} \dot{\tilde{p}}_{i}^{\mathrm{T}}(t-\tau) \tilde{p}_{i}(t-\tau)+2 \sum_{i=1}^{N_{i}} \tilde{q}_{i}^{\mathrm{T}}(t-\tau) \dot{\tilde{q}}_{i}(t-\tau)+\sum_{i=1}^{N_{i}} \sum_{j=1}^{N_{i}} a_{i j}\left[\tilde{q}_{i}^{\mathrm{T}}(0) \tilde{q}_{i}(0)\right. \\
& \left.-\tilde{q}_{i}^{\mathrm{T}}(t-\tau) \tilde{q}_{i}(t-\tau)\right]+\sum_{i=1}^{N_{i}} \sum_{j=1}^{N_{i}} a_{i j} \dot{\tilde{\varphi}}_{i j} \\
= & 2 k_{1} \sum_{i=1}^{N_{i}} \tilde{q}_{i}^{\mathrm{T}}(t-\tau) \tilde{p}_{i}(t-\tau)+\sum_{i=1}^{N_{i}} \sum_{j=1}^{N_{i}} a_{i j} \dot{\tilde{\varphi}}_{i j}+2 \sum_{i=1}^{N_{i}} \tilde{q}_{i}^{\mathrm{T}}(t-\tau)\left[-\sum_{j=1}^{N_{i}} a_{i j} \nabla_{\tilde{p}_{i j}} \dot{\tilde{\varphi}}_{i j}\right. \\
& \left.-k_{1} \tilde{p}_{i}(t-\tau)-k_{2} \tilde{q}_{i}(t-\tau)-\sum_{j=1}^{N_{i}} a_{i j}\left(\tilde{q}_{i}(t-\tau)-\tilde{q}_{j}(t-\tau)\right)\right] \\
& +\sum_{i=1}^{N_{i}} \sum_{j=1}^{N_{i}} a_{i j}\left[\tilde{q}_{j}^{\mathrm{T}}(0) \tilde{q}_{j}(0)-\tilde{q}_{j}^{\mathrm{T}}(t-\tau) \tilde{q}_{j}(t-\tau)\right] \\
= & \sum_{i=1}^{N_{i}} \sum_{j=1}^{N_{i}} a_{i j} \dot{\tilde{\varphi}}_{i j}-2 \sum_{i=1}^{N_{i}} \sum_{j=1}^{N_{i}} a_{i j} \tilde{q}_{i}^{\mathrm{T}} \nabla_{p_{i j}} \tilde{\varphi}_{i j}-2 k_{2} \sum_{i=1}^{N_{i}} \tilde{q}_{i}^{\mathrm{T}}(t-\tau) \tilde{q}_{i}(t-\tau) \\
& -2 \sum_{i=1}^{N_{i}} \sum_{i=1}^{N_{i}} a_{i j} \tilde{q}_{i}^{\mathrm{T}}(t-\tau) \tilde{q}_{i}(t-\tau)+2 \sum_{i=1}^{N_{i}} \sum_{i=1}^{N_{i}} a_{i j} \tilde{q}_{i}^{\mathrm{T}}(t-\tau) \tilde{q}_{j}(t-\tau) \\
& -\sum_{i=1}^{N_{i}} \sum_{i=1}^{N_{i}} a_{i j} \tilde{q}_{j}^{\mathrm{T}}(t-\tau) \tilde{q}_{j}(t-\tau)
\end{aligned}
$$

Due to the symmetry of the adjacency matrix $A$ and the artificial potential function $\varphi_{i j}$, we have

$$
\frac{\partial \varphi_{i j}\left\|\tilde{p}_{i j}\right\|}{\partial \tilde{p}_{i j}}=\frac{\partial \varphi_{i j}\left\|\tilde{p}_{i j}\right\|}{\partial \tilde{p}_{i}}=\frac{\partial \varphi_{i j}\left\|\tilde{p}_{i j}\right\|}{\partial \tilde{p}_{j}}
$$

Therefore, it obtains

$$
\begin{aligned}
\sum_{i=1}^{N_{i}} \sum_{j=1}^{N_{i}} a_{i j} \dot{\tilde{\varphi}}_{i j} & =\sum_{i=1}^{N_{i}} \sum_{j=1}^{N_{i}} a_{i j} \dot{\tilde{p}}_{i j}^{\mathrm{T}} \nabla_{\tilde{p}_{i j}} \tilde{\varphi}_{i j}=\sum_{i=1}^{N_{i}} \sum_{j=1}^{N_{i}} a_{i j}\left(\dot{\tilde{p}}_{i}^{\mathrm{T}} \nabla_{\tilde{p}_{i j}} \tilde{\varphi}_{i j}-\dot{\tilde{p}}_{i j}^{\mathrm{T}} \nabla_{\tilde{p}_{i j}} \tilde{\varphi}_{i j}\right) \\
& =\sum_{i=1}^{N_{i}} \sum_{j=1}^{N_{i}} a_{i j}\left(\dot{\tilde{p}}_{i}^{\mathrm{T}} \nabla_{\tilde{p}_{i}} \tilde{\varphi}_{i j}+\dot{\tilde{p}}_{i j}^{\mathrm{T}} \nabla_{\tilde{p}_{j}} \tilde{\varphi}_{i j}\right)=2 \sum_{i=1}^{N_{i}} \sum_{j=1}^{N_{i}} a_{i j} \dot{\tilde{p}}_{i}^{\mathrm{T}} \nabla_{\tilde{p}_{i}} \tilde{\varphi}_{i j} \\
& =2 \sum_{i=1}^{N_{i}} \sum_{j=1}^{N_{i}} a_{i j} \dot{\tilde{p}}_{i}^{\mathrm{T}} \nabla_{\hat{p}_{i j}} \tilde{\varphi}_{i j}=2 \sum_{i=1}^{N_{i}} \sum_{j=1}^{N_{i}} a_{i j} \tilde{q}_{i}^{\mathrm{T}} \nabla_{\tilde{p}_{i j}} \tilde{\varphi}_{i j}
\end{aligned}
$$

Substituting (20) into (18), yields

$$
\begin{aligned}
\dot{Q}= & -2 k_{2} \sum_{i=1}^{N_{i}} \tilde{q}_{i}^{\mathrm{T}}(t-\tau) \tilde{q}_{i}(t-\tau)-\sum_{i=1}^{N_{i}} \sum_{j=1}^{N_{i}} a_{i j} \tilde{q}_{i}^{\mathrm{T}}(t-\tau) \tilde{q}_{i}(t-\tau) \\
& -\sum_{i=1}^{N_{i}} \sum_{j=1}^{N_{i}} a_{i j}\left[\tilde{q}_{i}(t-\tau)-\tilde{q}_{j}(t-\tau)\right]^{\mathrm{T}}\left[\tilde{q}_{i}(t-\tau)-\tilde{q}_{j}(t-\tau)\right] \\
\leq & 0
\end{aligned}
$$

From (21), it can be seen that $Q$ is a non-increasing function. For notational convenience, we let $\tilde{p}=\operatorname{col}\left(\tilde{p}_{1}, \tilde{p}_{2}, \cdots, \tilde{p}_{N_{i}}\right) \in \square^{N_{i} \times n}$ and $\tilde{q}=\operatorname{col}\left(\tilde{q}_{1}, \tilde{q}_{2}, \cdots, \tilde{q}_{N_{i}}\right) \in \square^{N_{i} \times n}$, for any small time interval $t \square t+\Delta t, Q<Q_{0}$. From (17), we have $k_{1} \sum_{i=1}^{N_{i}} \tilde{p}_{i}^{\mathrm{T}} \tilde{p}_{i}<Q_{0}$, that is, $\tilde{p}_{i}^{\mathrm{T}} \tilde{p}_{i}<Q_{0} / k_{1}$, therefore, the distance between individual $i$ and its most correlated neighbor $f_{i}$ is not greater than $\sqrt{Q_{0} / k_{1}}$. Due to the connectivity of the subgroup, there exists a joint connected path 
from individual $i$ to $N_{i}$ in the small time interval $t \square t+\Delta t$, hence, the maximum distance between any two individual in the same subgroup is less than $\delta=\left(N_{i}-1\right) \sqrt{Q_{0} / k_{1}}$.

From above, it is also known that the set

$$
\Omega=\left\{\left[\tilde{p}^{\mathrm{T}}, \tilde{q}^{\mathrm{T}}\right] \in \square^{2 N_{i} n} \mid Q<Q_{0}\right\}
$$

is a compact invariant set. According to the LaSalle's invariant set principle, all the trajectories of individuals that start from $\Omega$ will converge to the largest invariant set inside the region $\Omega=\left\{\left[\tilde{p}^{\mathrm{T}}, \tilde{q}^{\mathrm{T}}\right] \in \square^{2 N_{i} n} \mid \dot{Q}=0\right\}$. Therefore, the velocity of individual $i$ will converge to that of its most correlated neighbor $f_{i}$, that is

$$
q_{i}=q_{f_{i}}
$$

Without loss of generality, we assume the $l$ th $\left(1 \leq l \leq N_{i}\right)$ individual in the subgroup is the only one that senses the external stimuli and it responses with fast maneuvering. According to (3), $l$ is the most correlated individual with largest information coupling degree. In the small time internal $t \square t+\Delta t$, there exists a joint connected path from individual $i$ to $l$, therefore, the velocities of all the individuals in the subgroup will converge to that of the $l$ th individual asymptotically, that is,

$$
q_{1}=q_{2}=\cdots=q_{l}
$$

where $q_{l}$ is the velocity of individual $l$ that senses the external stimuli.

Moreover, as the velocities of individuals in the same subgroup converge to that of the individual who senses external stimuli, the relative distance between different subgroups will increase with the divergence of individuals in different moving preferences, and ultimately the fission behavior occurs.

\section{Numerical Simulations}

To verify the effectiveness of the proposed fission control algorithm, numerical simulations are performed with 30 individuals in two dimensional plane.

\subsection{Simulation Setup}

The initial distributions of the 30 individuals are within the region of $15 \times 15 \mathrm{~m}^{2}$ and they are supposed to be connected. The sensing range of each individual is confined to $5 \mathrm{~m}$. The initial velocities are set with arbitrary directions and magnitudes within the range of $[0 \square 10] \mathrm{m} / \mathrm{s}$, their maximum and minimum velocities are constrained to $[-20 \square 20]^{\mathrm{T}} \mathrm{m} / \mathrm{s}$. Specifically, we let the time delay $\tau=0.5 \mathrm{~s}$. The other simulation parameters are chosen as $c^{*}=0.3, \Delta t=0.05 \mathrm{~s}, \varepsilon=0.2, k_{1}=k_{2}=1$.

Here, we consider the case where a cohesive flock separates into two clustered subgroups. We first let individuals that are with random initial positions and velocities aggregate and move in cohesive formation. Suppose that, at $t=6 \mathrm{~s}$, two conflict external stimuli with different directions act on two members of the flock (without loss of generality, we let them be individual 1 and individual 2, and then $g_{1}=g_{2}=1, g_{i}=0, i \neq 1,2$ ), and the desired velocities of individual 1 and individual 2 are $[-8 \square 0]^{\mathrm{T}} \mathrm{m} / \mathrm{s}$ and $[0 \square-8]^{\mathrm{T}} \mathrm{m} / \mathrm{s}$, respectively. 


\subsection{Simulation Results}

Based on the fission control algorithm (6), a self-organized fission behavior will occur under the conflict external stimuli. Simulation results are shown in Figure 2-Figure 4.
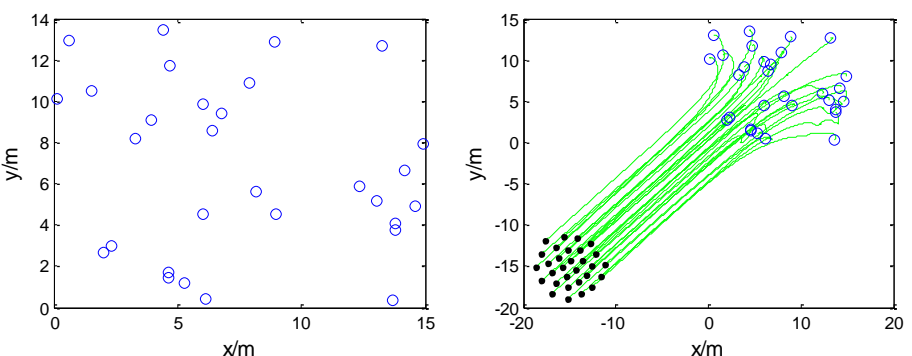

(a) $t=0 s$

(b) $t=5 s$
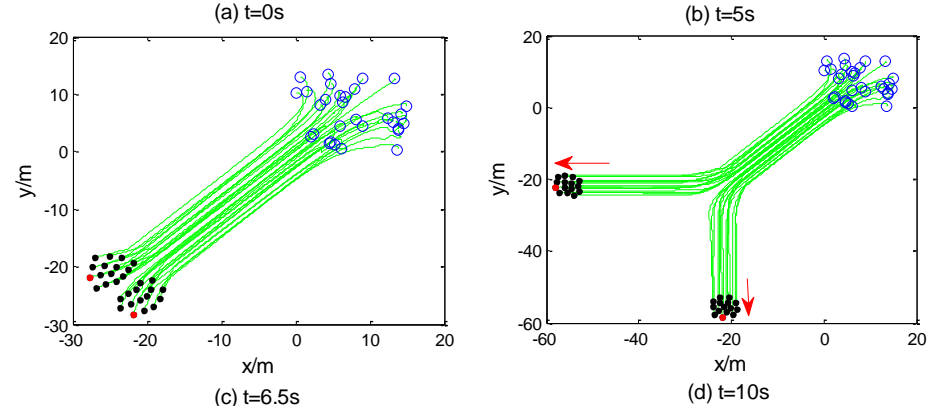

Figure 2. Snapshots of the Trajectories of Individuals during the Fission Process

Figure 2 (a)-(d) illustrate the moving trajectories of individuals during the fission process, where Figure 3 (a) is the initial distributions of individuals; Figure 3 (b) shows the aggregation and formation process of individuals form initial distribution to a cohesive group; Figure 3 (c) is the collective response of flocking system to external stimuli, two individuals that sense external stimuli change their movement to different directions and the flock tends to split under the fission control algorithm (6); Figure 3 (d) further demonstrates the fission process of flocking system and they finally form two clusters and the subgroups move in formation separately. 


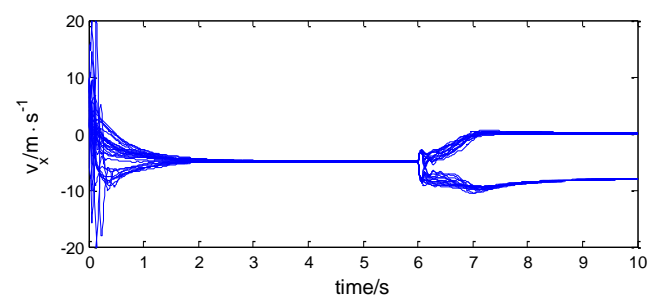

(a) Velocity of $x$-axis

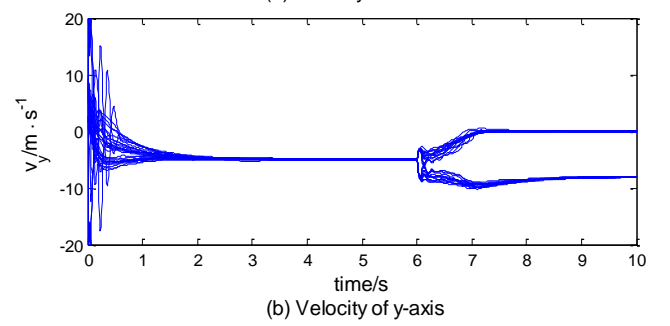

\section{Figure 3. Velocity of Individuals in the Fission Control of Flocking System with Time Delay}

Figure 3 (a) and (b) give the velocity curves of individuals during the simulation in both $x$ and $y$ axis. From which we can see that before external stimuli acts on the flock $(t<6 \mathrm{~s})$, individuals aggregate and move in formation with their velocities asymptotically converging to $[-5 \square-5]^{\mathrm{T}} \mathrm{m} / \mathrm{s}$; At $t=6 \mathrm{~s}$, under the influence of external stimuli, fission behavior occurs and the velocities of the two subgroups tend to that of the individuals who sense external stimuli. Eventually, the velocities of individuals in subgroup 1 converge to $[-8 \square 0]^{\mathrm{T}} \mathrm{m} / \mathrm{s}$ while the velocities of individuals in subgroup 2 converge to $[0 \square-8]^{\mathrm{T}} \mathrm{m} / \mathrm{s}$.

To better show the evolution of flocking system in the fission process, we introduce the order parameter ${ }^{\phi}$ to investigate the ordering of the flock

$$
\phi=\frac{1}{N_{i}}\left\|\sum_{i=1}^{N_{i}} \frac{q_{i}}{\left\|q_{i}\right\|}\right\|
$$

where $N_{i}$ is the number of individuals. Obviously, $\phi \in[0 \square 1]$. When $\phi \rightarrow 1$, the flock is said to be in an ordered state, otherwise, it is in a disordered state when $\phi \rightarrow 0$.

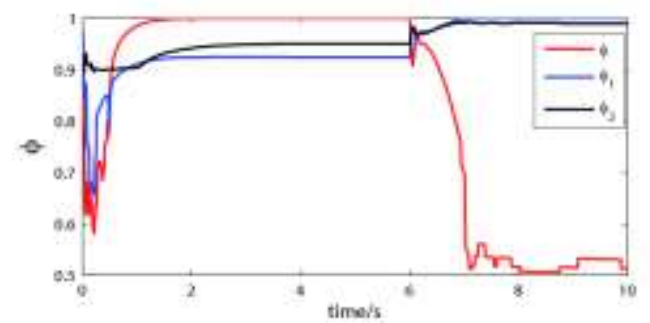

Figure 4. The Order Parameter of Flocking System in the Fission Process

Figure 4 demonstrates the order parameters of the flocking system in the fission process, where red line shows the order parameter $\phi$ of the whole flock, blue line and black line show 
the order parameters $\phi_{1}$ and $\phi_{2}$ of subgroup 1 and subgroup 2, respectively. From which we can see that before $t=6 \mathrm{~s}$, the flock first evolves from the random distribution to a relatively highly ordered state $\left(\phi, \phi_{1}, \phi_{2}>0.9\right)$. When environmental stimuli act on two members of the flock, the order parameter ${ }^{\phi}$ of the whole flock tends to decrease due to the bifurcation of the motion preferences of individuals. While the order parameters $\phi_{1}$ and $\phi_{2}$ of subgroup 1 and subgroup 2 finally reach a highly ordered state $\left(\phi_{1}, \phi_{2} \approx 1\right.$ ), demonstrating that the separated subgroups are able to move in stable formation after the fission behavior.

From the simulation results it can be concluded that the proposed fission control algorithm is capable of realizing the self-organized fission behavior of flocking system in the presence of time delay. When external stimuli act on a small portion of members, individuals in the same subgroup tend to move in a cohesive whole while individuals in different subgroup move towards different directions separately and the fission phenomenon will arise ultimately.

\subsection{Further Discussion}

To further investigate the influence of time delay on the performance of the proposed fission control algorithm, we increase the time delay to $\tau=1.5 \mathrm{~s}$. With the same parameters as the above simulation, we can get the following results.

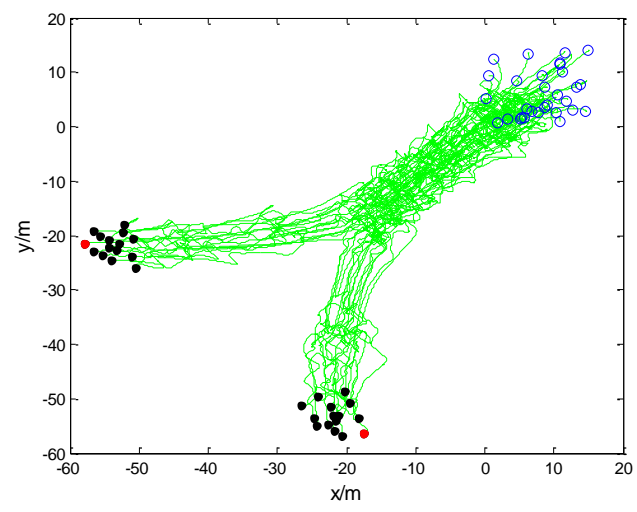

Figure 5. Trajectories of Individuals during the Fission Process with Time Delay $\tau=1.5 \mathrm{~s}$ 

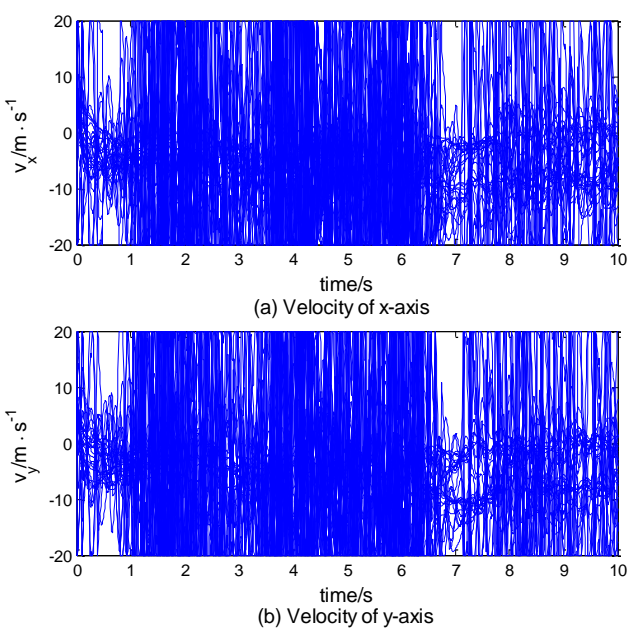

Figure 6. Velocity Curves of Individuals during the Fission Process with Time Delay $\tau=1.5 \mathrm{~s}$

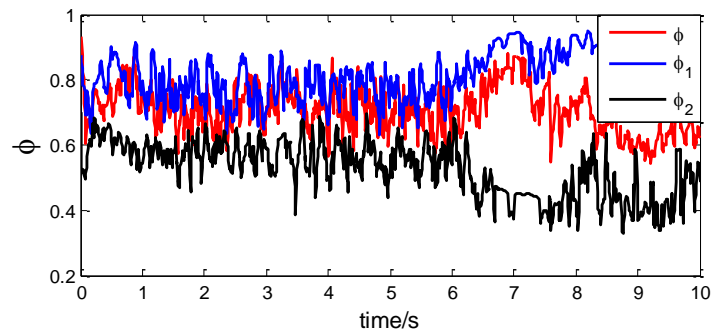

Figure 7. Order Parameters of Flocking System during the Fission Process with Time Delay $\tau=1.5 \mathrm{~s}$

From Figure $5 \sim$ Figure 7 , it can be seen that with the increase of time delay $\tau$, the proposed algorithm (6) can still realize the fission behavior, but the performance of the fission control algorithm decays significantly, where the trajectories of individuals tend to fluctuate violently (Figure 5) and the velocities of individual will not converge to the desired value (Figure 6). Moreover, the order parameters of the whole flock, subgroup 1 and subgroup 2 fluctuate and remain a relatively low value (Figure 7), demonstrating the disordering of the flock when the time delay is too big.

Therefore, the tolerable time delay upper bound that ensures the stability of fission control algorithm should be an important concern in our further research.

\section{Conclusions}

This paper studies the self-organized fission control problem for flocking system with time delay. Inspired by the fission principles of biological flocks, an information coupling degree based fission control schema is proposed. In consideration of the influence of time delay on the fission behavior, a distributed fission control algorithm with time delay is designed to achieve the spontaneous splitting of a cohesive flock into multiple clustered subgroups under conflict external stimuli. Both theoretical analysis and simulation results verify the effectiveness of the proposed fission control algorithm. 


\section{References}

[1] I. D. Couzin and M. E. Laidre, "Fission-fusion populations", Current Biology, vol. 19, no. 15, (2009), pp. 633-635.

[2] I. L. Bajec and F. H. Heppner, "Organized flight in birds", Animal Behavior, vol. 78, no. 4, (2009), pp. $777-$ 789.

[3] H.Duan and S. Liu, "Non-linear dual-mode receding horizon control for multiple unmanned air vehicles formation flight based on chaotic particle swarm optimization", IET Control Theory \& Applications, vol. 4, no. 11, (2010), pp. 2565-2578.

[4] H. M. La and W. Sheng, "Dynamic target tracking and observing in a mobile sensor network", Robotics and Autonomous Systems, vol. 60, no. 7, (2012), pp. 996-1009.

[5] G. Lee, N. Y. Chong, and H. Christensen, "Tracking multiple moving targets with swarms of mobile robots", Intelligent Service Robotics, vol. 3, no. 2, (2010), pp. 61-72.

[6] T. Vicsek and A. Zafeiris, "Collective motion", Physics Reports, vol. 517, no. 3, (2012), pp. 71-140.

[7] I. D. Couzin, J. Krause, N. R. Franks and S. A. Levin, "Effective leadership and decision-making in animal groups on the move", Nature, vol. 433, no. 7025, (2005), pp. 513-516.

[8] [8] M. Liu, P Yang, X. Lei and Y. Li, "Self-organized fission control for flocking system", Journal of Robotics, no. 321781, (2015), pp. 1-10.

[9] D. S. Morgan and I. B. Schwartz, "Dynamic coordinated control laws in multiple agent models", Physics Letters A, vol. 340, no. 1, (2005), pp. 121-131.

[10] M. Kumar, D. P. Grag and V. Kumar, "Segregation of heterogeneous units in a swarm of robotic agents", IEEE Transactions on Automatic Control, vol. 55, no. 3, (2010), pp. 743-748.

[11] Z. Chen, H. Liao and T. Chu, "Aggregation and splitting in self-driven swarms", Physica A, vol. 391, no. 15, (2012), pp. 3988-3994.

[12] X. Luo, S. Li and X. Guan, "Flocking algorithm with multitarget tracking for multi-agent systems", Pattern Recognition Letters, vol. 31, no. 9, (2010), pp. 800-805.

[13] H. Su, X. Wang and W. Yang, "Flocking in multi-agent systems with multiple virtual leaders", Asian Journal of control, vol. 10, no. 2, (2008), pp. 238-245.

[14] J. Li and A. H. Sayed, "Modeling bee swarming behavior through diffusion adaptation with asymmetric information sharing", EURASIP Journal on Advances in Signal Processing, no. 1, (2012), pp. 1-17.

[15] L. Jiang and R. Zhang, "Stable formation control of multi-robot system with communication delay", International Journal of Advanced Robotic Systems, vol. 9, (2012), pp. 1-10.

[16] R. Olfati-Saber and R. M. Murray, "Consensus problems in networks of agents with switching topology and time-delays", IEEE Transactions on Automatic Control, vol. 49, no. 9, (2004), pp. 1520-1533.

[17] J. Hu and Y. Hong, "Leader-following coordination of multiagent systems with coupling time delays", Physica A, vol. 374, no. 2, (2007), pp. 853-863.

[18] U. Munz, A. Papachristodoulou and F. Alllgower, "Delay dependent rendezvous and flocking of large scale multi-agent systems with communication delays", 47th IEEE Conference on Decision and Control, (2008), pp. 2038-2043.

[19] Z. Yang, Q. Zhang, Z. Jian and Z. Chen, "Flocking of multi-agents with time delay", International Journal of Systems Science, vol. 43, no. 11, (2012), pp. 2125-2134.

[20] D. J. Sumpter, "The principles of collective animal behavior", Philosophical Transactions of the Royal Society B: Biological Sciences, vol. 361, no. 1465, (2006), pp. 5-22.

[21] S. H. Lee, H. Pak and T. S. Chon, "Dynamics of prey-flock escaping behavior in response to predator's attack”, Journal of Theoretical Biology, vol. 240, no. 2, (2006), pp. 250-259.

[22] L. Conradt, J. Krause, I. Couzin and T. Poper, "Leading according to need in self-organizing groups", The American Naturalist, vol. 173, no. 3, (2009), pp. 304-312.

[23] S. Y. Tu and A. H. Sayed, "Effective information flow over mobile adaptive networks", 2012 3rd International Workshop on Cognitive Information Processing (CIP), (2012), pp. 1-6.

[24] C. W. Reynolds, "Flocks, herds and schools: A distributed behavioral model", ACM SIGGRAPH Computer Graphics, vol. 21, (1987), pp. 25-34.

[25] R. Olfati-Saber, "Flocking for multi-agent dynamic systems: Algorithms and theory", IEEE Transactions on Automatic Control, vol. 51, no. 3, (2006), pp. 401-420. 
International Journal of Control and Automation Vol. 10, No. 10 (2017) 